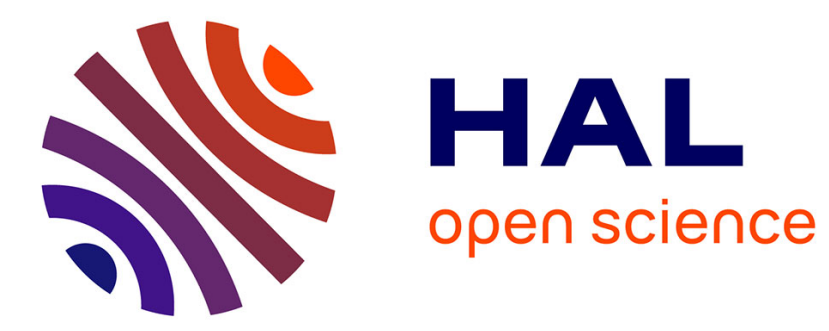

\title{
Measurements of some relaxed properties of point processes
}

Bernard Picinbono

\section{To cite this version:}

Bernard Picinbono. Measurements of some relaxed properties of point processes. IEEE Transactions on Instrumentation and Measurement, 2013, 62 (7), pp.2010-2019. hal-00838944

\section{HAL Id: hal-00838944}

https://hal-centralesupelec.archives-ouvertes.fr/hal-00838944

Submitted on 26 Jun 2013

HAL is a multi-disciplinary open access archive for the deposit and dissemination of scientific research documents, whether they are published or not. The documents may come from teaching and research institutions in France or abroad, or from public or private research centers.
L'archive ouverte pluridisciplinaire HAL, est destinée au dépôt et à la diffusion de documents scientifiques de niveau recherche, publiés ou non, émanant des établissements d'enseignement et de recherche français ou étrangers, des laboratoires publics ou privés. 


\title{
Measurements of some Relaxed Properties of Simulated Point Processes
}

\author{
Bernard Picinbono, Life Fellow, IEEE,
}

\begin{abstract}
This paper presents an algorithmic method for measurements of relaxed statistical properties of point processes (PP). In particular it makes it possible to determine the probability density function of the residual waiting time, which is the distance between an arbitrary origin and the first point of the PP posterior to this origin and also the probabilities of counting $n$ points of the PP in an interval open by an instant independent of the PP. These quantities are rarely accessible from a theoretical calculation. However, in the cases where this is possible, various experiments show quite good agreement with the theory.
\end{abstract}

\section{Index Terms}

Point processes, Simulation, Poisson processes, Time interval measurements, Counting, Simulations of point processes.

\section{INTRODUCTION}

Time point processes (PP) are used in numerous domains of Science, Engineering and Economics. There are especially appropriate to describe sequence of events arriving randomly in time appearing at a microscopic scale for example in the description of the emission of electrons or photons and also at a macroscopic scale in problems such as traffic congestion, random risks in insurances prediction and finance.

The mathematical theory of PPs is a well established field of Probability Theory and there exists an extensive literature on this subject going from very abstract and rigorous mathematical theory to more

Manuscript received August, 2012.

B. P. is with the Laboratoire des Signaux et Systèmes (L2S), a joint laboratory of the C.N.R.S. and the École Supérieure d'Électricité, Plateau de Moulon, 3 rue Joliot-Curie 91192, Gif sur Yvette, France. The L2S is associated with the University of Paris-Sud, France. E-mail: bernard.picinbono@1ss.supelec.fr, . 
applied approaches where practical problems are presented. In the first category we refer to [1] and in the latter to [2], [3], [4]. In this paper devoted to simulation and measurement problems we use more frequently the methods appearing in this second category.

In order to represent random physical events number of statistical models have been presented in the literature and a rather extensive presentation of these models appear in [2]. Among them the most important are certainly the stationary Poisson PPs defined by the fact that the time intervals between successive points, called lifetimes, are positive IID random variables (RV) with an exponential probability density function (PDF). Renewal PPs introduce also a large class of models important for the applications. They are defined by the fact that the lifetimes are positive RVs that are IID but with arbitrary PDFs. It is obvious that stationary Poisson PPs are also renewal processes. For various applications it is necessary to delete the assumption of stationarity and non stationary (or non homogeneous) Poisson PPs are the most important examples. Finally doubly stochastic Poisson PPs, or Poisson processes with a random density play a central role in Statistical Optics in order to describe the fluctuations of the electromagnetic field and various theoretical or experimental papers have been published on their properties. A good summary of this field of research can been found in [5], [6] and their use in Quantum Optics is summarized in [7], [8].

But PPs appearing in physical phenomena are sometimes too complicated to make it possible a clear mathematical analysis. It is then often much easier to work with simpler PPs obtained by computer simulations and we shall now present the most significant methods for this purpose. The simplest simulation procedures concern the renewal PPs. Indeed since a random distribution of points $T_{i}$ is completely determined by the sequence of lifetimes $X_{i}$ defined by $X_{i}=T_{i}-T_{i-1}$, and since for a renewal PP these RVs $X_{i}$ are IID, the simulation of such a PP is equivalent to that of a sequence of IID RVs of a given PDF. This is a classical problem well described in [9].

The simulation of non stationary and doubly stochastic Poisson processes is a much more difficult problem non still completely solved. The first attempt in this direction appeared in the fundamental paper [10] by using the so called thinning method well known for the simulation of RVs (see also [9]). This method was enlarged in [11]. But various other procedures have been proposed and applied in Statistical Optics [12], Biology [13] or in management problems [14], [15], [16]. Models of heartbeat PPs and their simulation have been presented in [17].

It is worth pointing out that the simulation procedures are not always limited to the generation of simulated PPs but can also be used for the simulation of some physical operations on such processes. One of the best examples is that of the dead time effect on PPs. This effect appears practically in every 
physical system and means that if the time interval between points is too small, some of these points are deleted. There are various different possible mechanisms of dead time effects. A rather elementary treatment of the two most important was presented in [2]. Their theoretical analysis is rather complicated and an approach where the PPs and the dead time effect are simulated is presented in [18], [19] for the so called output or input dead time effects which are quite different. Similarly the use of adaptive filtering for PPs models of heartbeat is presented in [20].

There are number of kinds of measurements in order to determine or to use the statistical properties of simulated PPs. For example in insurance or risks problems one is interested to the use of the methods of estimation or prediction well established in Signal Processing. Their transposition to PPs require some specific analysis [21]. In some applications in Physics or Astronomy direct measurements of some properties of PPs have a direct interpretation. This is for example the case in Statistical Optics where the measurements of the variance of the number of points of the PP measured in some time intervals is a practical mean to make a difference between classical or quantum optical fields [7], [8]. Indeed for the classical fields this variance is necessarily greater than the mean value, which justifies the precise measurements of this mean value and this variance. Measurements of moments or cumulants of order higher than two is also used in Astronomy [22]. Finally the importance of second order analysis with its consequences concerning the spectral properties of PPs justifies a specific interest in their measurements [23], [24].

But the most fundamental measurements concerning the statistical properties of PPs are those of counting probabilities and of time intervals distributions because they appear, as seen later, in the mathematical definition of these processes. In physical and engineering literature the basic devices for such measurements are counters and time to amplitude converters (TAC). A counter is a linear filter with an impulse response $h(t)$ equal to 1 in the interval $[0, T]$ and to 0 otherwise. If its input is a sequence of pulses at random time instants $T_{i}$, its output is the signal $s(t)=\sum h\left(t-T_{i}\right)$ sometimes called shot noise of the PP (see p. 321 of [25]) and it results from the specific value of $h(t)$ that $s(t)$ is equal to the number $N(t, T)$ of random points $T_{i}$ appearing in the interval $[t-T, t]$. From the processing of a great number of values of $N(t, T)$ we can deduce the counting probabilities. On the other hand by using a TAC system we can convert time intervals in pulses and the statistical analysis of these pulses can yield their probability density function which appears in the mathematical definition of PPs. A great number of such results obtained some time ago in Statistical Optics are reported in [5].

The purpose of this paper is to transpose these methods in the case of simulated PPs in which the input data on the process is the sequence of distances $X_{i}$ between successive points. Because of the structure 
of these inputs the use of the shot noise signal is no longer the simplest way to reach the counting probabilities and a quite different approach appears necessary. For this purpose let us first recall some fundamental definitions and notations on PPs.

A point process (PP) is a random distribution of points in some space. In everything that follows this space is the time axis, which introduces the expression of of time PP. There exist two main ways to describe the statistical properties of PPs. Consider a PP $\mathcal{P}$ and let us note $T_{i}$ its random points, that are time instants classed in an increasing order $\left(T_{i}<T_{i+1}\right)$. The intervals $X_{i}$ between successive points $\left(X_{i}=T_{i}-T_{i-1}\right)$ are called the lifetimes, or sometimes interevents times. These quantities are positive random variables $(\mathrm{RV})$ and it is clear that $\mathcal{P}$ is described as well by the sequences of $T_{i}$ or by the sequences of $X_{i}$. This implies that the statistical properties of $\mathcal{P}$ are entirely described by those of the $X_{i} \mathrm{~s}$ which can be considered as the values of a positive discrete time signal. For this description we can apply all the standard methods used in random signal theory. In this case we use the expression of of time intervals description of a PP.

The second approach is called counting description. Consider $M$ arbitrary non-overlapping time intervals $\Delta_{k}$ and let $N_{k}$ be the random number of points $T_{i}$ of $\mathcal{P}$ appearing in $\Delta_{k}$. If for any $M$ and for each $M$ for any set on intervals $\Delta_{k}$ we know the statistical properties of the set of RVs $N_{k}$, we also have a complete description of the statistical properties of $\mathcal{P}$. In this case we use the term of counting approach which comes from the fact that the random numbers $N_{k}$ are physically obtained by a counting procedure.

The simplest, and probably the most important, example of PP is the stationary Poisson process. In this case the RVs $X_{i}$ are positive IID random variables with an exponential distribution and the $N_{k} \mathrm{~s}$ are independent RVs with a Poisson distribution.

The relation between these two approaches to the description of a PP is a well documented question. It appears in the books indicated above and in [26], [27] and in the references herein.

Let us now introduce the concept of relaxed properties. Consider an arbitrary time $\theta$ which is not a point of $\mathcal{P}$. Let $T(\theta)$ be the first point of $\mathcal{P}$ posterior to $\theta$. The positive time interval $T(\theta)-\theta$ is called the residual lifetime, or also the residual waiting time (RWT). It is obviously an RV and in principle its statistical properties can be deduced from those defining the PP but the calculations are often very complicated. It is clear, however, that if $\mathcal{P}$ is stationary, these properties do not depend on $\theta$. The residual waiting time is the simplest example of a relaxed property in which a time instant $\theta$ that does not have any relation with the PP $\mathcal{P}$ is introduced. In contrast, the lifetime, which is the interval between two successive points of the PP, is called a triggered property. It is clear that for practical application the 
relaxed properties have the same importance as the triggered ones. For example, in the analysis of the behaviour of a switching system for telephone calls, the customer is more interested in his waiting time than in the interval between successive calls, even if these two properties are closely interconnected.

The same distinction can be introduced in the case of counting properties. If the intervals $\Delta_{k}$ introduced above have no relation with the PP $\mathcal{P}$ we talk about relaxed counting. On the other hand, if these intervals have, for example, the form $\left[T_{i}, T_{i}+T\right]$, which means that the beginning of the interval is a point of $\mathcal{P}$, we talk about triggered counting. In the case where $\mathcal{P}$ is stationary, the statistical properties of the number of points $N$ recorded in $[\theta, \theta+T]$ does not depend on $\theta$, but it is clear, and we shall verify this point below, that for the same value of the length $T$ these statistical properties of $N$ have no reason to be the same if $\theta=T_{i}$ or not, i.e. in the triggered or the relaxed cases. The best example appears for PPs with dead-time effects. If all the points of $\mathcal{P}$ belonging to the intervals $\left[T_{i}, T_{i}+D\right]$, where $D$ is the dead-time constant, are erased, we obtain a new PP in which no point can appear in intervals such as $\left[T_{i}, T_{i}+D\right]$ while this property has no reason to be true for intervals such $[\theta, \theta+D]$, where $\theta$ is not a point of the PP, (see [18], [19] and references herein).

There is, however, a case where this distinction between relaxed and triggered properties disappears. This is the case of stationary Poisson processes mentioned above. Indeed one of the characteristic properties of such processes is the fact that they have no memory, which suppresses the difference between triggered and relaxed properties.

Measurements of relaxed properties are in general more complicated than those of triggered ones. This appears clearly in the case of renewal processes, which, after the Poisson processes, are the most important for practical applications. Such PPs are defined by the fact that the intervals between successive points (lifetimes) are IID positive RVs. Consequently the statistical properties of these PPs are entirely defined by the distribution function (DF) of all the lifetimes. It is clear that the triggered properties of such processes result from certain properties of the sums of IID random variables, which is a rather classic problem of probabilities. On the other hand, measurement and calculation of relaxed properties such as the residual waiting times are in general much more complicated and, in some cases, almost impossible. It is the purpose of this paper to present some measurement methods for these properties and when calculations are affordable to compare their results with those obtained with our measurement methods in order to evaluate their performances. 


\section{PRinciples of the Algorithms}

Let $\mathcal{P}_{S}$ be a stationary PP, the index $S$ meaning that it is the signal that we want to analyze. Its ordered sequence of instants is noted $\left\{s_{i}\right\}$. In order to obtain its relaxed properties it is necessary to have at our disposal another stationary and ordered sequence of time instants $t_{i}$ independent of $\mathcal{P}_{S}$ They are the instants opening its analysis according to the procedure described below. These instants constitute the analysis PP called $\mathcal{P}_{A}$. The only property of $\mathcal{P}_{A}$ required at this step is to be independent of $\mathcal{P}_{S}$ and we shall discuss below the relative interest of the various possible choices.

To each point $t_{i}$ of $\mathcal{P}_{A}$ we associate the point of $\mathcal{P}_{S}$ noted $s_{j(i)}$ which is the last point of $\mathcal{P}_{S}$ anterior to $t_{i}$, which means that $\left(t_{i}-s_{j(i)}\right)>0$ and $\left(t_{i}-s_{j(i)+1}\right)<0$. It is then characterized by the fact that it is the only point of the sequence $\left\{s_{j}\right\}$ such that the product $\left(t_{i}-s_{j}\right)\left(t_{i}-s_{j+1}\right)$ is negative. This property can be used for its calculation. Indeed for each $t_{i}$ we can calculate the products $\left(t_{i}-s_{j}\right)\left(t_{i}-s_{j+1}\right), 1 \leq j \leq M$, where $M$ is the number of possible values of $j$ and $j(i)$ is the only value of $j$ among possible $M$ for which this product is negative. This procedure however requires very long calculation times because there are of the order of $M^{2}$ products to calculate and if $M \sim 10^{6}$, as in most of our following experiments, this can require more than one hundred hours of calculations using standard computers.

It is then more appropriate to introduce a recursive procedure which can significantly reduce the calculation time. This results from the following principle. Suppose that $s_{j(i)}$ is known and consider the difference $d_{n}(i+1)=t_{i+1}-s_{j(i)+n}$. It is clear that $j(i+1)=j(i)+n$ if and only if $d_{n}(i+1)>0$ and $d_{n+1}(i+1)<0$. This yields the following:

$$
j(i+1)=j(i)+\sum_{n=1}^{\infty} n F_{n}(i),
$$

with

$$
F_{n}(i)=u\left[d_{n}(i+1)\right] v\left[d_{n+1}(i+1)\right]
$$

where $u(x)$ is the unit step function equal to 1 if $x>0$ and to -1 otherwise, and $v(x)=u(-x)$. The advantage of (1) seems a priori weak, because of the presence of a series of an infinite number of terms. It appears however that this number can in practice be very small. In particular when the densities $\lambda$ and $\nu$ of the PPs $\mathcal{P}_{S}$ and $\mathcal{P}_{A}$ respectively satisfy $\lambda<<\nu$, which is a very common situation, a short calculation shows that there is practically only one term corresponding to $n=1$ in the series of (1), and for each set of experiments presented below we have introduced a procedure ensuring that replacing the series by a sum of a finite number of terms does not introduce an error in the calculation of (1).

Once the sequence $j(i)$ is known, it can be used for the measurements of some relaxed properties of the PP $\mathcal{P}_{S}$. Let us begin by the analysis of the residual waiting time (RWT). 
The RWT associated with each instant $t_{i}$ of the PP $\mathcal{P}_{A}$ is, from the definition of the sequence $s_{j(i)}$, the quantity $s_{j(i)+1}-t_{i}$. It is a positive random variable (RV), and if the sequence of the $t_{i}$ s contains $M_{A}$ values, there are also $M_{A}$ values of this RV. Assuming that the PPs $\mathcal{P}_{S}$ and $\mathcal{P}_{A}$ are stationary and ergodic, we can deduce from the normalized histogram of these values the probability density function (PDF) of the RWT which is in general rather difficult to obtain from a theoretical calculation, except for some cases discussed below. For a less extensive analysis it is possible to limit the measurements to the determination of the mean and the variance of the RWT.

Consider now the counting analysis. The problem is to determine the statistics of the number of points of $\mathcal{P}_{S}$ in time intervals of duration $T$ and open by the points $t_{i}$ of $\mathcal{P}_{A}$.

Let $D_{n}(i)$ be the time interval between $t_{i}$ and the $n^{\text {th }}$ point of $\mathcal{P}_{S}$ posterior to $t_{i}$. It results from the definition of $s_{j(i)}$ that $D_{n}(i)=s_{j(i)+n}-t_{i}$, in such a way that the intervals $D_{n}(i)$ are known as soon as the $s_{j(i)} \mathrm{s}$ are known. It is clear that there are $n$ points of $\mathcal{P}_{S}$ in the interval $\left[t_{i}, t_{i}+T\right]$ (relaxed counting) if and only if $D_{n}(i)<T$ and $D_{n+1}(i)>T$. This leads to introduce the function $G_{n}(i)=u\left[D_{n+1}(i)-T\right] v\left[D_{n}(i)-T\right]$, where $u($.$) and v($.$) have been defined after (2). We note then$ that $G_{n}(i)$ takes only the values 0 or 1 , and furthermore that $G_{n}(i)=1$ if and only if there are $n$ points in $\left[t_{i}, t_{i}+T\right]$. As the expected value of a RV taking only the values 0 or 1 is equal to the probability that this $\mathrm{RV}$ is equal to 1 , we deduce that the probability $p_{n}$ that there are $n$ relaxed points in the interval $\left[t_{i}, t_{i}+T\right]$ is the expected value $\mathrm{E}\left[G_{n}(i)\right]$. Finally it results from our assumptions of stationarity and ergodicity that this expected value is the time average, or $\left(1 / M_{A}\right) \sum_{1}^{M_{A}} G_{n}(i)$, where $M_{A}$ is the number of time instants $t_{i}$ introduced for the analysis. Finally it is clear that if $n=0$, the corresponding relaxed probability $p_{0}$ is simply the ensemble, or time, mean value of $u\left[D_{1}(i)-T\right]$.

Note finally that if the instants $t_{i}$ are chosen among the $s_{i}$ the previous algorithms yield either the lifetime statistics or the triggered counting probabilities.

\section{EXPERIMENTAL RESULTS}

\section{A. Principles of the Experiments}

We shall now present various experimental results for the analysis of PPs obtained by applying the previous algorithms. The starting point is the definition of the $\operatorname{PP} \mathcal{P}_{S}$ subject of this analysis. As indicated in the introduction, $\mathcal{P}_{S}$ can be defined by the sequence $X_{i}$ of the distances between successive points which are positive RVs, or constitute a positive discrete time signal. This signal is obtained either from a physical experiment or from a computer simulation. We use this second procedure exclusively in 
everything that follows. In other words we realize computer measurements on simulated data, but the computer measurements can also be carried out with real physical data.

Our purpose is to measure some properties of $\mathcal{P}_{S}$ from a sequence of $M$ realizations $s_{i}$ of its lifetimes $S_{i}$. In order to use the algorithms discussed in the previous section we must introduce an analysis stationary $\mathrm{PP} \mathcal{P}_{A}$. The only requirement concerning this PP is the relation introduced above between its density $\nu$ and the density $\lambda$ of $\mathcal{P}_{S}$. In all the experiments presented below we have chosen $\nu=10 \lambda$. On the other hand we have verified that the statistical properties of $\mathcal{P}_{A}$ do not play any role in the results of the analysis, in such a way that we have chosen the simplest PP possible defined by the fact that the lifetimes are constant and equal to $1 / \nu$. The other important point to define is the number $M_{A}$ of values of $\mathcal{P}_{A}$ chosen for the experiment. We must adapt this number to the number $M$ of values of $\mathcal{P}_{S}$. Indeed it results clearly from the structure of the calculation of $j(i)$ that if $M_{A}$ is too small a large number of values of $\mathcal{P}_{S}$ are not taken into account, and on the contrary if $M_{A}$ is too high many values of $\mathcal{P}_{A}$ are useless. Since the mean duration of the sequence of $\mathcal{P}_{S}$ and $\mathcal{P}_{A}$ are $M / \lambda$ and $M_{A} / \nu$ respectively, we have chosen the compromise $M_{A}=(\nu / \lambda) M$. Finally it is appropriate to choose values of $M$ sufficiently large to obtain a fairly good statistical precision when measuring time averaging. In most of our experiment, $M$ is of the order of $10^{6}$. Greater values are sometimes necessary, but at the expense of much longer experiment time.

\section{B. Poisson Processes}

In order to verify the behaviour of our method we shall begin with the simplest and most common example of PP which is the stationary Poisson process. Such a process is defined by the fact that the positive RVs $X_{i}$ are IID and with an exponential distribution. There are various standard programs for the generation of a sequence of such RVs, the most common consisting in realizing a specific nonlinear transformation of a sequence of IID random variables uniformly distributed in the interval $[0,1]$. More precisely let $W_{i}$ be such a sequence. It is then easy to verify that the RVs $X_{i}$ defined by $X_{i}=-(1 / a) \ln \left(W_{i}\right)$ are IID and with the PDF $p(x)=a \exp (-a x)$. Their mean value and variance are $1 / a$ and $1 / a^{2}$ respectively. It is well known that the PDFs of the RWT and of the lifetimes of a stationary Poisson process are the same and equal to $p(x)$. In Fig. 1 we present experimental results of measurements of these PDFs obtained from normalized histograms either of the $X_{i}$ or of the results of the measurements of the RWT by using the algorithms described above. In this figure, as in all the others that follow, the continuous curves correspond to the theoretical PDF while the points represent the values of the measurements of these PDS from a standard processing of experimental histograms. For 
this experiment the value chosen for the parameter $a$ of the DDP is $a=1$. As expected the experimental points are very well located on the continuous curve which with the choice of semilogarithmic coordinates is simply a straight line. This figure clearly shows that the PDF of the RWT is $\exp (-x)$ which means, as predicted by the theory, that the RWT and lifetime have the same exponential PDF.

Let us now consider the case of relaxed counting experiments. They are realized for two values of $T$, $T=0.5$, and $T=1$. The theory predicts that the random number of relaxed points in these intervals are Poisson RVs with mean $m=\lambda T$. Since $\lambda=1$, which results from the choice of the value $a=1$ for the coefficient of the exponential distribution of the lifetimes, these mean values are 0.5 or 1 for the two experiments reported in Table 1. In this table we present the theoretical values of the counting probabilities $p_{n}$ given by $p_{n}=\exp (-m)\left(m^{n} / n !\right)$. The last two columns of the table indicate the values of the sum of the $p_{n} \mathrm{~s}$ appearing on the same line and the corresponding mean values. We observe a fairly good agreement between the theoretical results and those measured with our algorithms, which indicates a good performance of the method introduced above.

Table 1. Relaxed Counting Probabilities for Poisson Processes.

\begin{tabular}{cccccccccccc} 
& $p_{0}$ & $p_{1}$ & $p_{2}$ & $p_{3}$ & $p_{4}$ & $p_{5}$ & $p_{6}$ & $p_{7}$ & $p_{8}$ & Sum & mean \\
\hline$T=0.5$ & & & & & & & & & & & \\
\hline $\mathrm{TH}$ & 0.6065 & 0.3033 & 0.0758 & 0.0126 & 0.0016 & 0.0002 & 0 & 0 & 0 & 1.0000 & 0.5000 \\
\hline $\mathrm{EXP}$ & 0.6069 & 0.3030 & 0.0755 & 0.0128 & 0.0017 & 0.0002 & 0.0000 & 0 & 0 & 1.0000 & 0.5000 \\
\hline$T=1$ & & & & & & & & & & & \\
\hline $\mathrm{TH}$ & 0.3679 & 0.3679 & 0.1839 & 0.0613 & 0.0153 & 0.0031 & 0.0005 & 0.0001 & 0.0000 & 1.0000 & 1.0000 \\
\hline $\mathrm{EXP}$ & 0.3688 & 0.3671 & 0.1831 & 0.0616 & 0.0157 & 0.0031 & 0.0005 & 0.0001 & 0.0000 & 1.0000 & 1.0000 \\
\hline
\end{tabular}

\section{Erlang(2) Processes}

Let $U$ and $V$ be two IID random variables defined by their common exponential PDF $p(x)=$ $a \exp (-a x)$ and $X$ be the sum $X=U+V$. Its PDF is $f(x)=a^{2} x \exp (-a x)$ and the corresponding mean value is $2 / a$. An Erlang(2) PP is a renewal PP (lifetimes are a sequence of IID RVs) in which the PDF of the lifetimes is $p_{L}(x)=f(x)$. Its density $\lambda$ is clearly $\lambda=a / 2$. It is rather simple to calculate the PDF $p_{R W T}(x)$ of the RWT and the results expressed with the density $\lambda$ are

$$
p_{L}(x)=4 \lambda^{2} x \exp (-2 \lambda x), p_{R W T}(x)=\lambda(1+2 \lambda x) \exp (-2 \lambda x) .
$$


Furthermore a more complicated calculation yields the relaxed counting probabilities given by $p_{0}=$ $(1+\mu / 2) \exp (-\mu)$ and for $k \geq 1$,

$$
p_{k}=(1 / 2) \exp (-\mu) \frac{\mu^{2 k+1}}{(2 k-1) !}\left[1+\frac{\mu}{k}+\frac{\mu^{2}}{2 k(2 k+1)}\right],
$$

where $\mu=2 \lambda T$.

It is clear that it is very easy to simulate an Erlang(2) PP because it only requires the generation of two IID positive exponential RVs. In other words the complexity of its simulation is on the same order as that of a Poisson process.

In the following experiments we have generated samples of an Erlang(2) process of density $\lambda=1$, which yields $a=2$. As previously these samples are used for time or counting measurements.

In Fig. 2 we present results of measurements of the PDFs of the lifetime and of the RWT of Erlang(2) PPs. As in the previous figure, the continuous curves correspond to the theoretical values while the points are coming from experimental normalized histograms. But the great difference with the case of the Poisson process is that the PDFs $p_{L}(x)$ and $p_{R W T}(x)$ of the lifetime and the RVWT respectively are quite different. This clearly appears in Fig. 2 in which we also observe that the experimental points are located with quite a good degree of precision on the theoretical curve. So, in this case where calculations are also possible, these results confirm the validity of the measurements method.

Let us now present the results of counting probabilities measurements. They are listed in Table 2, presented in the same manner as Table 1. For three values of the time duration $T$ we present the theoretical and measured values of the counting probabilities. We observe that the results are quite similar, which again confirms the validity of the measurement procedure presented above.

Table 2. Relaxed Counting Probabilities for Erlang(2) Processes.

\begin{tabular}{cccccccccccc} 
& $p_{0}$ & $p_{1}$ & $p_{2}$ & $p_{3}$ & $p_{4}$ & $p_{5}$ & $p_{6}$ & $p_{7}$ & $p_{8}$ & Sum & Mean \\
\hline$T=0.5$ & & & & & & & & & & & \\
\hline $\mathrm{TH}$ & 0.5518 & 0.3985 & 0.0475 & 0.0021 & 0.0000 & 0.0000 & 0.0000 & 0.0000 & 0.0000 & 1.0000 & 0.5000 \\
\hline $\mathrm{EXP}$ & 0.5522 & 0.3981 & 0.0476 & 0.0021 & 0.0000 & 0 & 0 & 0 & 0 & 1.0000 & 0.4996 \\
\hline$T=1$ & & & & & & & & & & & \\
\hline $\mathrm{TH}$ & 0.2707 & 0.4962 & 0.1985 & 0.0318 & 0.0027 & 0.0001 & 0.0000 & 0.0000 & 0.0000 & 1.0000 & 0.9993 \\
\hline $\mathrm{EXP}$ & 0.2708 & 0.4967 & 0.1979 & 0.0316 & 0.0028 & 0.0001 & 0.0000 & 0 & 0 & 1.0000 & 1.0000 \\
\hline$T=2$ & & & & & & & & & & & \\
\hline $\mathrm{TH}$ & 0.0549 & 0.2808 & 0.3712 & 0.2121 & 0.0662 & 0.0129 & 0.0017 & 0.0002 & 0.0000 & 1.0000 & 2.0000 \\
\hline $\mathrm{EXP}$ & 0.0554 & 0.2809 & 0.3715 & 0.2111 & 0.0663 & 0.0130 & 0.0017 & 0.0002 & 0.0000 & 1.0000 & 1.9986 \\
\hline
\end{tabular}




\section{Erlang(3) Processes}

These PPs are natural extensions of those presented in the previous section. Instead of starting from two RVs $U$ and $V$, we add a third one $W$ independent of the others and with the same exponential distribution and we consider the sum $X=U+V+W$. An Erlang(3) PP is a renewal PP in which the distances between successive points are IID random variables with the distribution of $X$. It is easy to find the PDF $p_{L}(x)$ of the lifetime and a little less easy to obtain $p_{R W T}(x)$, PDSF of the RWT. The results are

$$
p_{L}(x)=(27 / 2) \lambda^{3} x^{2} \mathrm{e}^{-3 \lambda x}, p_{R W T}(x)=\lambda\left(1+3 \lambda x+(9 / 2) \lambda^{2} x^{2}\right) \mathrm{e}^{-3 \lambda x},
$$

where, as in (3), $\lambda$ is the density of the PP. In order to obtain an Erlang(3) PP of density $\lambda=1$ from simulations of three sequences of IID exponential RVs with PDF $p(x)=a \exp (-a x)$, it suffices to take $a=3$. Experimental results presented on the same manner as those of Fig. 2 appear on Fig. 3. As in the previous figures, the position of the experimental points on the theoretical curves deduced from (5) shows an excellent agreement between experiment and theory.

Let us now consider counting experiments. After rather complex algebra we obtain the values of the relaxed counting probabilities $p_{n}$ of a Erlang(3) PP given by $p_{o}=(1 / 3) \mathrm{e}^{-m}\left[3+2 m+m^{2} / 2\right]$ and for $n>0$

$$
p_{n}=(1 / 3) \mathrm{e}^{-m} m^{3 n-2} T_{n}(m)
$$

where $m=3 \lambda T$ and

$$
T_{n}(m)=\frac{1}{(3 n-2) !}+\frac{2 m}{(3 n-1) !}+\frac{3 m^{2}}{(3 n) !}+\frac{2 m^{3}}{(3 n+1) !}+\frac{m^{4}}{(3 n+2) !} .
$$

Experimental results for an $\operatorname{Erlag}(3)$ PP of density $\lambda=1$ compared with theoretical values given by these equations appear in Table 3.

Table 3. Relaxed Counting Probabilities for Erlang(3) Processes.

\begin{tabular}{cccccccccccc} 
& $p_{0}$ & $p_{1}$ & $p_{2}$ & $p_{3}$ & $p_{4}$ & $p_{5}$ & $p_{6}$ & $p_{7}$ & $p_{8}$ & Sum & Mean \\
\hline$T=0.5$ & & & & & & & & & & & \\
\hline $\mathrm{TH}$ & 0.5299 & 0.4405 & 0.0292 & 0.0004 & 0.0000 & 0.0000 & 0.0000 & 0.0000 & 0 & 1.0000 & 0.5000 \\
\hline $\mathrm{EXP}$ & 0.5298 & 0.4404 & 0.0294 & 0.0004 & 0.0000 & 0 & 0 & 0 & 0 & 1.0000 & 0.4996 \\
\hline$T=1$ & & & & & & & & & & & \\
\hline $\mathrm{TH}$ & 0.2240 & 0.5688 & 0.1907 & 0.0159 & 0.0005 & 0.0000 & 0.0000 & 0.0000 & 0 & 1.0000 & 1.0000 \\
\hline $\mathrm{EXP}$ & 0.2241 & 0.5682 & 0.1912 & 0.0160 & 0.0005 & 0.0000 & 0 & 0 & 0 & 1.0000 & 1.0007 \\
\hline$T=2$ & & & & & & & & & & & \\
\hline $\mathrm{TH}$ & 0.0273 & 0.2667 & 0.4385 & 0.2186 & 0.0443 & 0.0044 & 0.0002 & 0.0000 & 0 & 1.0000 & 2.0000 \\
\hline $\mathrm{EXP}$ & 0.0280 & 0.2649 & 0.4384 & 0.2203 & 0.0440 & 0.0043 & 0.0002 & 0.0000 & 0 & 1.0000 & 2.0013 \\
\hline
\end{tabular}


As for the other examples presented above this table shows a quite good correspondence between the theoretical values of the counting probabilities and those obtained from experiments using the algorithms discussed in the previous sections. These results open the use of these algorithms in the cases where theoretical calculations are almost impossible, as seen in the example discussed in the next section.

\section{E. Renewal Processes with Triangular Distribution}

As indicated above, a renewal PP is entirely defined by the PDF common to all its lifetimes which also are IID random variables. In this section we assume that this PDF $p_{X}(x)$ is triangular with mean value equal to 1 . This implies that $p_{X}(x)=u(x) v(x-1) x+u(x-1) v(x-2)(2-x)$, where $u$ and $v$ are defined after (2). Its distribution function (DF) $F_{X}(x)$ is then equal to

$$
F_{X}(x)=u(x) v(x-1) x^{2} / 2+u(x-1) v(x-2)\left(1-2 x+x^{2} / 2\right)+u(x-2) .
$$

It is shown in pps. 8 and 54 of [2] that for a renewal PP the PDF of the residual waiting time $X$ is given by

$$
p_{R W T}(x)=\left(1 / m_{X}\right)\left[1-F_{X}(x)\right] .
$$

The simulation of the renewal PP characterized by the DF (8) is especially simple. Indeed it is obvious that (8) is the DF of the sum of two IID random variables uniformly distributed in the interval $[0,1]$. Applying our method of measurement to a sequence of such RVs we can measure the PDFs of the lifetime and of the RWT. The results appear in Fig 4. The continuous curves correspond to the theoretical PDFs and the points to the experimental measurement of these PDFs. We observe on this figure an excellent correspondence between experimental and theoretical results.

The situation of the counting measurements is quite different. Indeed it is almost impossible to obtain in closed form the mathematical expression of the counting probabilities, as it was the case for the other experiments displayed in this paper. This is the reason why Table 4 presents only experimental measurements realized in the same conditions as in the other experiments. 
Table 4. Relaxed Counting Probabilities for Renewal PP With tRiangular PDF.

\begin{tabular}{cccccccccccc} 
& $p_{0}$ & $p_{1}$ & $p_{2}$ & $p_{3}$ & $p_{4}$ & $p_{5}$ & $p_{6}$ & $p_{7}$ & $p_{8}$ & Sum & Mean \\
\hline$T=0.5$ & & & & & & & & & & & \\
\hline $\operatorname{EXP}$ & 0.5209 & 0.4587 & 0.0201 & 0.0003 & 0 & 0 & 0 & 0 & 0 & 1.0000 & 0.4997 \\
\hline$T=1$ & & & & & & & & & & & \\
\hline $\operatorname{EXP}$ & 0.1668 & 0.6752 & 0.1499 & 0.0079 & 0.0002 & 0.0000 & 0 & 0 & 0 & 1.0000 & 0.9994 \\
\hline$T=2$ & & & & & & & & & & & \\
\hline $\operatorname{EXP}$ & 0 & 0.2340 & 0.5578 & 0.1849 & 0.0220 & 0.0012 & 0.0000 & 0.0000 & 0 & 1.0000 & 1.9987 \\
\hline
\end{tabular}

It is finally interesting to compare the measured counting probabilities $p_{n}$ of the four PPs discussed above for the same value $T=1$ of the counting interval. Since these four PPs have the same density $\lambda=1$, they also introduce the same mean value of the relaxed number of points in this interval. The results appear in Table 5.

Table 5. Relaxed Counting Probabilities for the Previous PPs for $T=1$.

\begin{tabular}{cccccccccccc} 
& $p_{0}$ & $p_{1}$ & $p_{2}$ & $p_{3}$ & $p_{4}$ & $p_{5}$ & $p_{6}$ & $p_{7}$ & $p_{8}$ & Sum & Mean \\
\hline Poisson & 0.3688 & 0.3671 & 0.1831 & 0.0616 & 0.0157 & 0.0031 & 0.0005 & 0.0001 & 0.0000 & 1.0000 & 1.0000 \\
\hline Erlang(2) & 0.2708 & 0.4967 & 0.1979 & 0.0316 & 0.0028 & 0.0001 & 0.0000 & 0 & 0 & 1.0000 & 1.0000 \\
\hline Erlang(3) & 0.2241 & 0.5682 & 0.1912 & 0.0160 & 0.0005 & 0.0000 & 0 & 0 & 0 & 1.0000 & 1.0007 \\
\hline Triang. & 0.1668 & 0.6752 & 0.1499 & 0.0079 & 0.0002 & 0.0000 & 0 & 0 & 0 & 1.0000 & 0.9994 \\
\hline
\end{tabular}

\section{ACKNOWLEDGEMENTS}

The author thanks Malcolm Scott for helpful suggestions concerning the English of this paper.

\section{REFERENCES}

[1] D. J. Daley and D. A. Vere-Jones, An Introduction to the Theory of Point Processes, New York: Springer, 2008.

[2] D. R. Cox and V. Isham, Point Processes, London, U.K.: Chapman and Hall, 1980.

[3] D. L. Snyder and M. I. Miller, Random Point Processes in Time and Space, New York: Springer, 1991.

[4] S. Lowen and M. Teich, Fractal-Based Point Processes, New-York: Wiley, 2005.

[5] B. Saleh, Photoelectron Statistics, Berlin: Springer, 1978.

[6] B. Saleh and M. Teich, Fundamentals of Photonis, Hoboken, N. J.: Wiley, 1991.

[7] B. Picinbono and C. Bendjaballah, "Characterization of nonclassical optical fields by photoelectron statistics," Phys. Rev. A, 71, , pp. 013812, 1-12, 2005.

[8] B. Picinbono and C. Bendjaballah, "Some properties of point processes in statistical optics," Phys. Rev. A, 82, , pp. 063811, $1-8,2010$. 
[9] L. Devroye, Non-Uniform Random Variate Generation, Berlin: Springer, 1986. accessible at http: //www.nrbook.com/devroye/

[10] P. A. W. Lewis and S. Shedler, "Simulation of nonhomogeneous Poisson processes by thinning," Naval Research Logistics Quarterly , 26, , pp. 403-413, 1979.

[11] Y. Ogata, "On Lewis simulation method for point processes," IEEE Trans. Inform. Theory, vol. IT-27:, pp. 23-31, 1981.

[12] V. Apanasovich, E. Novikov, “Computer simulation of photoevents," Jour. Modern Optics, vol. 40, pp. 213-218, 1993.

[13] S. Shinomoto, Y. Tsubo, "Modeling spiking behavior of neurons with time-dependent Poisson processes," Physical Review, vol. E 64, pp. 041910 1-13, 2001.

[14] K. Burnecki, W. Hardle, R. Weron, “Simulation of risk Processes,” Encyclopedia of Actuarial Science, vol. 3, pp. 1564-1570, 2004.

accessible at http://www.im.pwr.wroc.pl/ hugo/publ/HSC_03_4_BurneckiHaerdleRWeron.pdf

[15] S. Harrod and W. Kelton, "Numerical methods for realizing nonstationary Poisson processes with piecewise-constant instantaneous-rate functions," Simulation, vol. B82-3, pp. 147-157, 2006.

[16] K. Giesecke, M. Kakavand, M. Mousavi, "Exact simulation of point processes with stochastic intensities," Operations Research, vol. 59, pp. 1233-1245, 2011.

accessible at

http://www.stanford.edu/dept/MSandE/cgi-bin/people/faculty/giesecke/pdfs/projection.pdf

[17] Z. Chen Z., E. N. Brown E. N. and R. Barbieri, "Characterizing nonlinear heartbeat dynamics within a point process framework,” IEEE Trans. Biomed. Eng., 57, , pp. 1335-1347, 2010.

[18] B. Picinbono, "Output Dead-Time in Point Processes," Communications in Statistics.Simulation and Computation, vol. 38, pp. 2198-2213, 2009.

[19] B. Picinbono, “Temps mort d'entrée dans les processus ponctuels," Traitement du signal, vol. 24, pp. 411-428, 2007.

[20] R. Barbieri and E. Brown, "Analysis of heart beat dynamics by point process adaptive filtering," IEEE Trans. Biomed. Eng., 53, , pp. 4-12, 2006.

[21] C. Andrieu, "Bayesian deconvolution of noisy filtered point processes," IEEE Trans. Signal Proc., 49, pp. 134 - $146,2001$.

[22] R. Thacker and H.. Couchman, "A new fast parallel statistical measurement technique for computational cosmology," International Journal of High Performance Computing and Networking, 4, pp. 303-310, 2006.

[23] B. Picinbono, “ Measurements of second-order properties of point processes," IEEE Trans. Instrumentation and Measurement, vol. 57, pp. 548-555, 2008.

[24] G. Adelfio and F. Paik Schoenberg, "Point process diagnostics based on weighted second-order statistics and their asymptotic properties," Ann. Inst. Stat. Math. , 61, pp. 929-948, 2009.

[25] Picinbono B., Random Signals and Systems, Englewood Cliffs, N.J. : Prentice Hall, 1993.

[26] J. A. McFadden, "On the lengths of intervals in a stationary point process," J. Roy. Statist. Soc., vol. B-24, $364382,1962$.

[27] B. Picinbono, “Time intervals and counting in point processes," IEEE Trans. Information Theory, vol. IT-50, pp. 1336-1340, June 2004. 


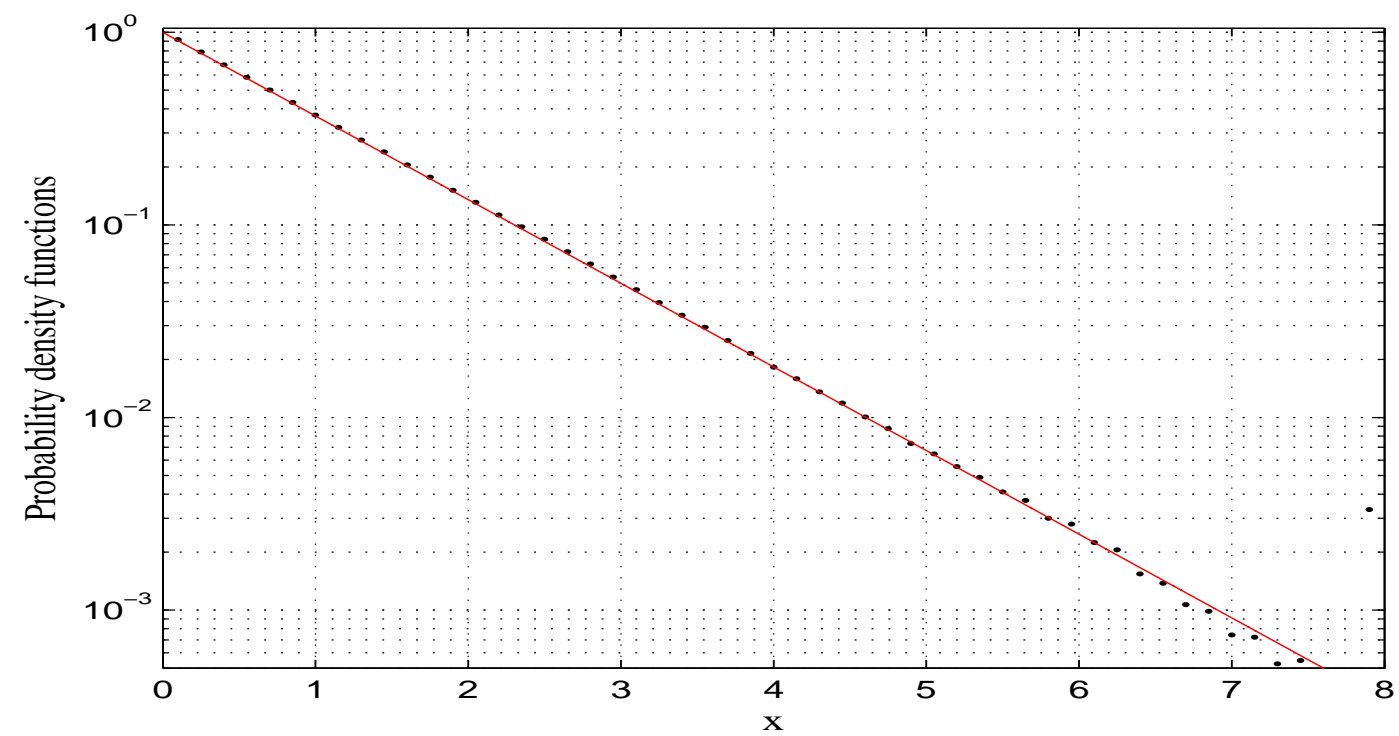

Fig. 1. Probability density functions $p(x)$ of the residual waiting time of a Poisson process. Points: experiments with simulated data, continuous curves: theory or $p(x)=\exp (-x)$.

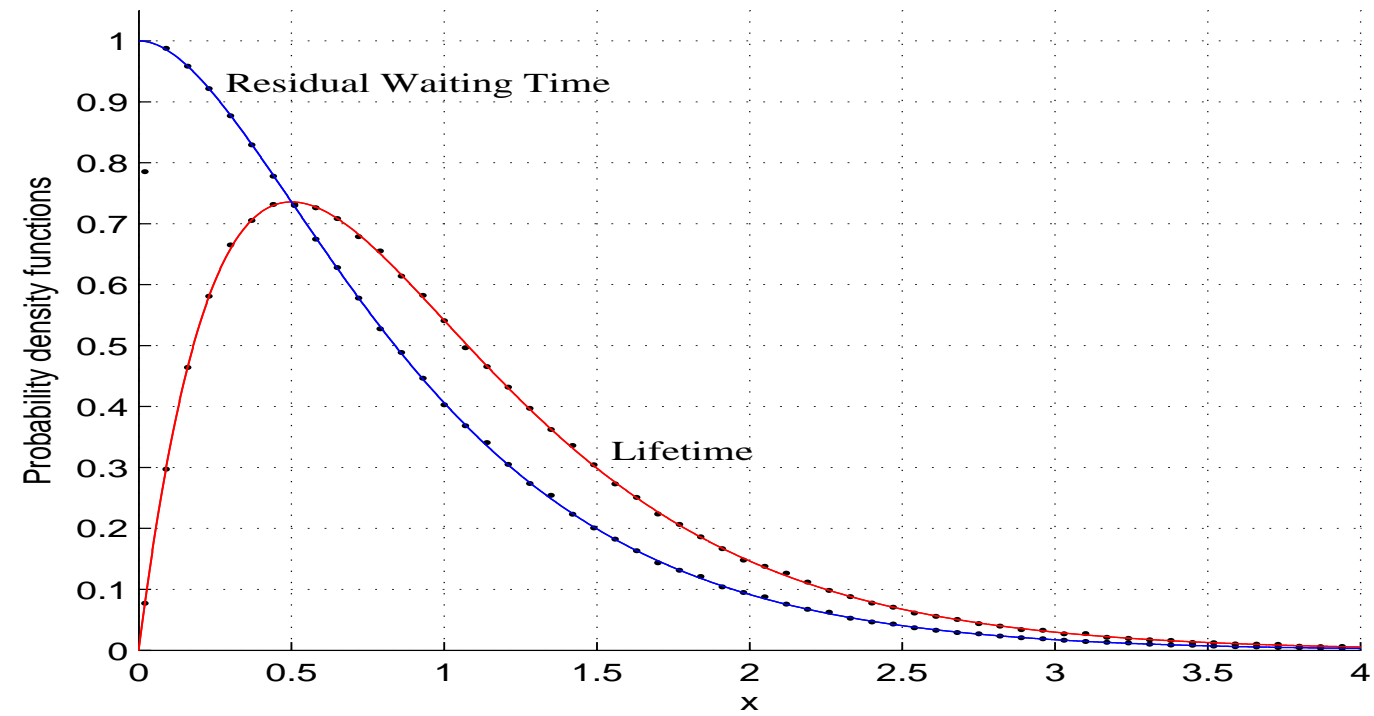

Fig. 2. Probability density functions of RWT and of lifetime for an Erlang(2) PP. Points: experiment with simulated data, continuous curve: theory or $p_{R W T}(x)=\lambda(1+2 \lambda x) \exp (-2 \lambda x), p_{L}(x)=4 \lambda^{2} x \exp (-2 \lambda x), \lambda=1$. 


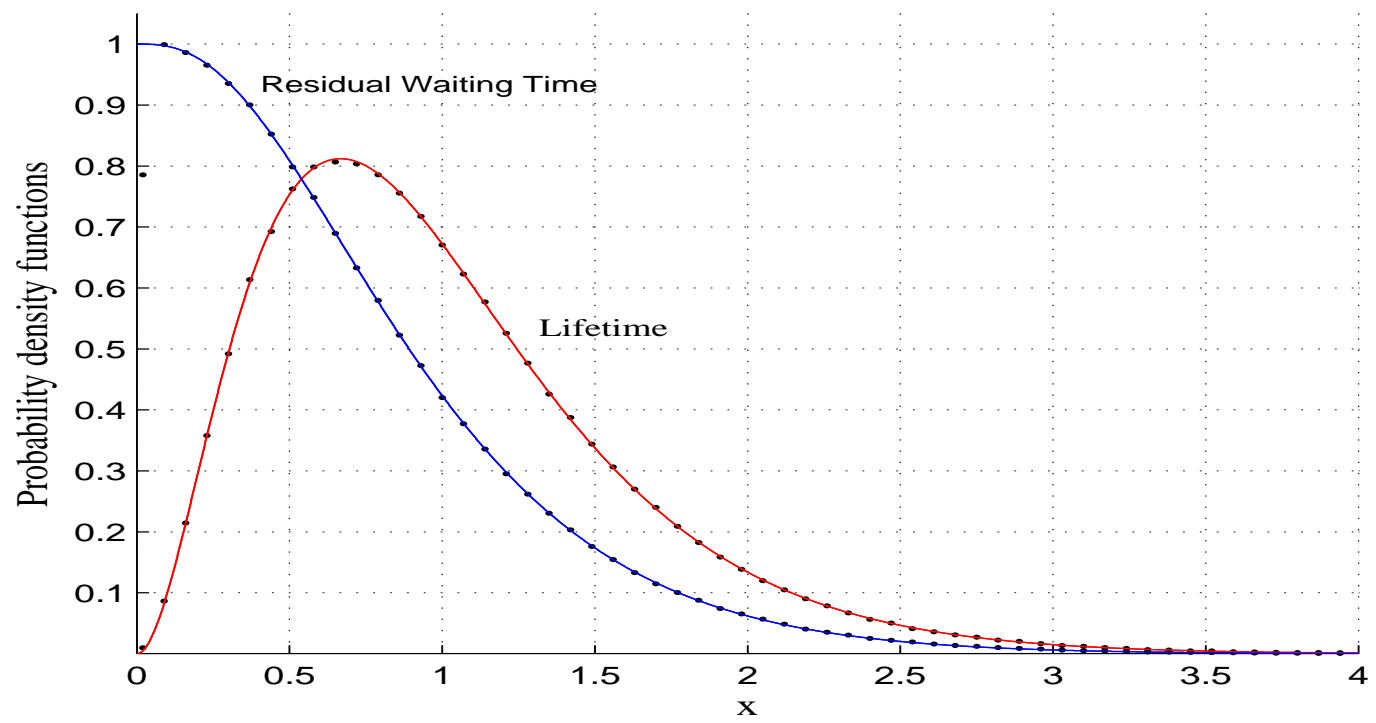

Fig. 3. Probability density functions of RWT and of lifetime for an Erlang(3) PP. Points: experiment with simulated data, continuous curve: theory given by (5)

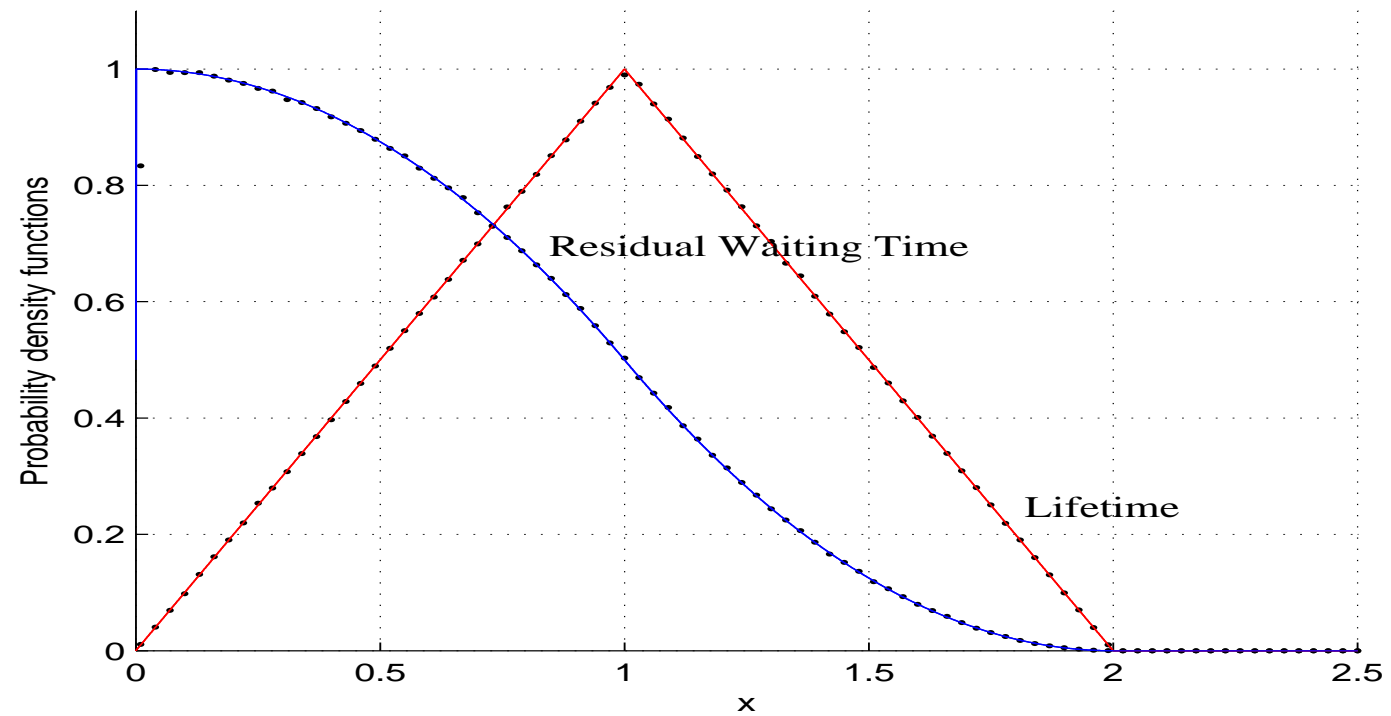

Fig. 4. Probability density functions of RWT and of lifetime for a renewal PP with triangular lifetime. Points: experiment with simulated data, continuous curve: theory given by (8) and (9). 